\title{
ON THE RATIONAL HOMOTOPY LIE ALGEBRA OF A FIXED POINT SET OF A TORUS ACTION
}

\author{
CHRISTOPHER ALLDAY ${ }^{1}$ AND VOLKER PUPPE
}

\begin{abstract}
Let $X$ be a simply connected topological space, and let $\mathscr{L}_{*}(X)$ be its rational homotopy Lie algebra. Suppose that a torus acts on $X$ with fixed points, and suppose that $F$ is a simply connected component of the fixed point set. If $\mathscr{L}_{*}(X)$ is finitely presented and if $F$ is full, then it is shown that $\mathscr{L}_{*}(F)$ is finitely presented, and that the numbers oi generators and relations in a minimal presentation of $\mathscr{L}_{*}(F)$ do not exceed the numbers of generators and relations (respectively) in a minimal presentation of $\mathscr{L}_{*}(X)$. Various other related results are given.
\end{abstract}

1. Introduction. For a simply connected space $X$ let $\mathscr{L}_{*}(X)$ denote the rational homotopy Lie algebra of $X$. (See [14], for example.) Thus $\mathscr{L}_{n}(X)=\pi_{n+1}(X) \otimes Q$, where $Q$ is the field of rational numbers. Now suppose that a torus group $T$ acts on $X$ with nonempty fixed point set $X^{T}$, and let $F$ be a component of $X^{T}$. In [4], under certain conditions, we proved a theorem relating the numbers of generators and relations in a minimal presentation of $\mathscr{L}_{*}(X)$ to those in a minimal presentation of $\mathscr{L}_{*}(F)$. In this paper we give a new proof of an extended version of this theorem. The new proof has a number of advantages over the proof in [4]. For example, in [4] we assumed that $\operatorname{dim}_{Q} \mathscr{L}_{*}(X)$ was finite, whereas here we need assume only that $\mathscr{L}_{*}(X)$ is finitely presented. The new proof also gives a method for computing the numbers of generators and relations in the presentations of $\mathscr{L}_{*}(X)$ and $\mathscr{L}_{*}(F)$. In addition, a crucial tool of the proof, the equivariant Milnor-Moore-Quillen spectral sequence (see §3), may have other uses in the rational cohomology and homotopy theory of torus group actions.

Before stating the main theorem, we need to list some conditions. The first set of conditions (1.1) is standard; the second set (1.2) is more particular to our theorem.

1.1 Conditions. $\operatorname{dim}_{Q} H^{*}(X ; Q)<\infty$, and either

(A) $X$ is compact, or

(B) $X$ is paracompact, $X$ has finite rational sheaf dimension $\left(c d_{Q}(X)<\infty\right)$, and the number of connective isotropy subgroups is finite.

The last condition in (B), which is not needed in (A), states that $\left\{T_{x}^{0}: x \in X\right\}$ is finite, where $T_{x}^{0}$ is the identity component of the isotropy subgroup $T_{x}$ at $x$. This condition is, of course, redundant if $T=S^{1}$, the circle group.

Received by the editors April 12, 1984 and, in revised form. October 10, 1985.

1980 Mathematics Subject Classification. Primary 57S99, 55Q91; Secondary 55P62.

Key words and phrases. Torus actions, rational homotopy Lie algebra, generators and relations, cup length.

${ }^{1}$ Research partially sponsored by NSF Grant No. MCS79-02036-A01.

O1986 American Mathematical Society $0002-9947 / 86 \$ 1.00+\$ .25$ per page 
1.2 Conditions. (i) ' $X$ and $F$ are 1-connected,

(ii) $X$ and $F$ are agreement spaces.

That is, the natural transformation $\bar{H}^{*}(; Q) \rightarrow H^{*}(; Q)$ between rational Alexander-Spanier cohomology and rational singular cohomology is an isomorphism on $X$ and $F$. (Spaces having the homotopy type of $\mathrm{CW}$-complexes, for example, are agreement spaces.)

(iii) $F$ is full. (See Definition 3.2.)

Our main theorem is

1.3 Theorem. Suppose $X$ and $F$ satisfy Conditions 1.1 and 1.2. Suppose further that $\mathscr{L}_{*}(X)$ is finitely presented (as a Lie algebra) and let $L\left(W_{X}\right) \rightarrow L\left(V_{X}\right) \rightarrow \mathscr{L}_{*}(X) \rightarrow 0$ be a minimal presentation, where $L$ is the free graded Lie algebra functor. Then $\mathscr{L}_{*}(F)$ is finitely presented; and if $L\left(W_{F}\right) \rightarrow L\left(V_{F}\right) \rightarrow \mathscr{L}_{*}(F) \rightarrow 0$ is a minimal presentation, then

(1) $\operatorname{dim}_{Q}\left(V_{F}\right) \leqslant \operatorname{dim}_{Q}\left(V_{X}\right)$,

(2) $\operatorname{dim}_{Q}\left(W_{F}\right) \leqslant \operatorname{dim}_{Q}\left(W_{X}\right)$, and, indeed,

(3) c.i.d. $\left(\mathscr{L}_{*}(F)\right) \leqslant$ c.i.d. $\left(\mathscr{L}_{*}(X)\right)$,

where c.i.d. $\left(\mathscr{L}_{*}(X)\right)=\operatorname{dim}_{Q}\left(W_{X}\right)-\operatorname{dim}_{Q}\left(V_{X}\right)$.

We also prove a result on iterated Whitehead products and degrees of nilpotency (Theorem 4.6).

1.4 Remarks. (i) Conditions 1.2(i) and (ii) are undesirable. Some results follow from the proof in $\$ 4$ without them. If (i) does not hold, one can use pseudo-dual rational homotopy co-Lie algebra structures. (See [2, 8 and 15], for example.) If (ii) does not hold, one can use the Alexander-Spanier-Sullivan-de Rham theory of [3]. We shall not pursue these lines here, however.

(ii) Condition 1.2(iii) is needed. It is analogous to the cohomology condition " $X$ is totally nonhomologous to zero (TNHZ)". (It is, however, independent of that condition.) Examples 4.4 and 4.5 show that the theorem can fail if $F$ is not full.

(iii) The condition " $\operatorname{dim}_{Q} H^{*}(X ; Q)<\infty$ " in 1.1 , which implies that $\operatorname{dim}_{Q} H^{*}(F ; Q)<\infty$, could be replaced by the conditions " $H^{*}(X ; Q)$ and $H^{*}(F ; Q)$ have finite type."

(iv) The cohomology analogue of Theorem 1.3 is due to T. Chang ([6], but see also [11]) for part (2), and to L. Avramov and V. Puppe (see [13]) for part (3). Very simple elementary proofs of the cohomology theorem have been found by both authors of this paper independently ([13], for example).

The authors wish to thank the organizers of the conference "Méthodes d'Algèbre Homotopique en Topologie" (C.I.R.M., June 1982), J.-M. Lemaire, and J.-C. Thomas, for their generous hospitality. It was at the C.I.R.M. that our collaboration began.

2. Remarks on equivariant rational homotopy. In this section we give a rather technical extension of a result of [1] and [2]. As in [1] let $A$ be a commutative differential $\mathbf{Z}_{2}$-graded $K$-algebra, where $K$ is a field of characteristic zero. Suppose that $A$ has an augmentation $\varepsilon: A \rightarrow K$. Let $m=\operatorname{ker}(\varepsilon)$, the augmentation ideal. 
2.1 Definition. Let $Q_{n, k}(A)=m^{n} / m^{n+k}$, for $n$ and $k$ positive integers, and let $\pi_{n, k}(A)=H\left(Q_{n, k}(A)\right)$, the homology of $Q_{n, k}(A)$ with respect to the differential induced by the differential on $A$. (Thus, in the notation of [1], $Q_{1,1}(A)=Q(A)$ and $\pi_{1,1}(A)=\pi(A)$.)

Now let $U$ be the $\mathbf{Z}_{2}$-graded $K$-vector space with basis $\{v, w\}$, with $\operatorname{deg}(v)=0$, $\operatorname{deg}(w)=1$. Let $S(U)=K[v] \otimes \Lambda(w)$ be the free commutative $\mathbf{Z}_{2}$-graded $K$-algebra generated by $U$. Suppose we are given $u \in A$ with $\operatorname{deg}(u)=0, d u=0$, and $\varepsilon(u)=1$. Form $A \otimes S(U)$ and extend the differential $d$ and augmentation $\varepsilon$ by the rules $d v=0, d w=u v-1, \varepsilon(v)=1, \varepsilon(w)=0$. Then we have the following extension of [1, Remark 3.6]. (We omit the proof, which is straightforward.)

2.2 Lemma. The inclusion $A \rightarrow A \otimes S(U)$ induces a weak equivalence $Q_{n, k}(A) \rightarrow$ $Q_{n, k}(A \otimes S(U))$. That is, the inclusion induces an isomorphism

$$
\pi_{n, k}(A) \rightarrow \pi_{n, k}(A \otimes S(U)) .
$$

Now let $T$ be a torus and let $X$ be a $T$-space satisfying Conditions 1.1. Let $T \rightarrow E_{T} \rightarrow B_{T}$ be the universal principal $T$-bundle, $X \rightarrow X_{T} \rightarrow B_{T}$ the associated $X$-bundle, $R=H^{*}\left(B_{T} ; Q\right)$, and $K=R_{(0)}$, the localization of $R$ with respect to the multiplicative set of nonzero homogeneous elements. Let $\mathscr{M}(X)$ denote the Sullivan minimal model of $X$. Suppose that the fixed point set $X^{T}$ is nonempty, and let $F$ be a component of $X^{T}$.

By the Grivel-Halperin-Thomas theorem (see [8], for example) $\mathscr{M}\left(X_{T}\right)=R \otimes$ $\mathscr{M}(X)$ with the differential being twisted, in general; and the inclusion $F \rightarrow X$ induces an $R$-linear augmentation $\varepsilon(F): \mathscr{M}\left(X_{T}\right) \rightarrow \mathscr{M}\left(F_{T}\right)=R \otimes \mathscr{M}(F) \rightarrow R$. (Note that the differential in $R \otimes \mathscr{M}(F)$ is not twisted, since $F$ is fixed, and so $F_{T}=B_{T} \times F$.) Let $\mu: \mathscr{M}(F) \rightarrow Q$ be the augmentation of $\mathscr{M}(F)$, and let $\bar{\mu}$ : $R \otimes \mathscr{M}(F) \rightarrow R$ be induced by $\mu$ (i.e., $\bar{\mu}=$ id $\otimes \mu$ ). Thus $\varepsilon(F)=\bar{\mu} \varphi(F)$, where $\varphi(F)$ is the map $\mathscr{M}\left(X_{T}\right) \rightarrow \mathscr{M}\left(F_{T}\right)$, which is well defined up to homotopy.

2.3 Definition. Let $m=\operatorname{ker}(\varepsilon(F))$. Set $Q_{(T, F, n, k)}(X)=m^{n} / m^{n+k}$, and $\Pi_{(T, F, n, k)}^{*}(X)=H\left(Q_{(T, F, n, k)}(X)\right)$. (Thus in the notation of [2], $Q_{(T, F, 1,1)}(X)=$ $Q_{(T, F)}(X)$, and $\Pi_{(T, F, 1,1)}^{*}(X)=\Pi_{(T, F)}^{*}(X)$.) Also let $\Pi_{(n, k)}^{*}(F)=\pi_{n, k}(\mathscr{M}(F))$ with respect to $\mu$.

The methods of [1] and [2], together with 2.2 above, imply the following proposition.

2.4 Proposition. The homomorphism $\varphi(F)^{*}: \Pi_{(T, F, n, k)}^{*}(X) \rightarrow \Pi_{(T, F, n, k)}^{*}(F)=R$ $\otimes \Pi_{(n, k)}^{*}(F)$, induced by $\varphi(F)$, becomes an isomorphism when localized with respect to the multiplicative set of nonzero homogeneous elements in $R$.

3. The equivariant Milnor-Moore-Quillen spectral sequence. Let $X$ be a connected space of finite rational type (i.e., $H^{*}(X ; Q)$ has finite type), let $\mathscr{M}(X)$ be the minimal model of $X, \varepsilon: \mathscr{M}(X) \rightarrow Q$ the augmentation, and $m=\operatorname{ker}(\varepsilon)$ the augmentation ideal. Then the filtration $\mathscr{M}(X) \supseteq m \supseteq m^{2} \supseteq \cdots \supseteq m^{n} \supseteq m^{n+1} \supseteq \cdots$ 
gives rise to a spectral sequence $E(X)_{r}^{*, *} \Rightarrow H^{*}(X ; Q)$. According to Felix and Halperin [7], if $X$ is simply connected, then, for $r \geqslant 2$, this spectral sequence is dual to the Milnor-Moore spectral sequence $E(X)_{*, *}^{r} \Rightarrow H_{*}(X ; Q)$ in which $E(X)_{*, *}^{2}=$ $\operatorname{Tor}_{*, *}^{\Omega}(Q, Q)$, where $\Omega=H_{*}(\Omega X ; Q)$ [10, Théorème 2.2]. Equally, according to Tanré [16], if $X$ is simply connected, then, for $r \geqslant 1, E(X)_{r}^{*, *}$ is dual to the Quillen spectral sequence [14, 6.9]. To see that the Quillen and Milnor-Moore spectral sequences coincide from the $E_{2}$-term on, one need only observe that Quillen uses a Koszul complex construction, whereas Moore uses the bar and cobar constructions. (This comparison was made in [5].)

Now, for a torus $T$, suppose that $X$ is a simply connected $T$-space satisfying Conditions 1.1 and 1.2. As in $\S 2$ suppose that $X^{T} \neq \varnothing$, let $F$ be a component of $X^{T}$, and let $\varphi(F): \mathscr{M}\left(X_{T}\right) \rightarrow \mathscr{M}\left(F_{T}\right)$ and $\varepsilon(F): \mathscr{M}\left(X_{T}\right) \rightarrow R$ be the induced map and augmentation, respectively. Let $m=\operatorname{ker}(\varepsilon(F))$. Then the filtration $\mathscr{M}\left(X_{T}\right) \supseteq m$ $\supseteq m^{2} \supseteq \cdots \supseteq m^{n} \supseteq m^{n+1} \supseteq \cdots$ gives rise to a spectral sequence $E(T, F ; X)_{r} \Rightarrow$ $H_{T}^{*}(X ; Q)$. We shall call this spectral sequence the equivariant Milnor-MooreQuillen spectral sequence (EMMQSS) of $(T, F ; X)$.

$\varphi(F)$ induces a homomorphism of spectral sequences

$$
\varphi(F)_{r}^{* *}: E(T, F ; X)_{r}^{*, *} \rightarrow E(T, F ; F)_{r}^{*, *} \cong R \otimes E(F)_{r}^{*, *} .
$$

For each $p \geqslant 0, E(T, F ; X)_{r}^{p, *}$ is an $R$-module; and with the notation of $\S 2$,

$$
E(T, F ; X)_{0}^{p, *}=Q_{(T, F, p, 1)}(X) \text { and } E(T, F ; X)_{1}^{p, *}=\Pi_{(T, F, p, 1)}^{*}(X) .
$$

Thus 2.4 implies the following proposition.

3.1 Proposition. For $r \geqslant 1, \varphi(F)_{r}^{* * *}$ becomes an isomorphism when localized with respect to the multiplicative set of nonzero homogeneous elements in $R$.

The following definition of fullness can be shown to be equivalent to that given in [2], where $F$ is said to be full if $\Pi_{(T, F)}^{*}(X)$ is a free $R$-module.

3.2 Definition. $F$ is said to be full in $X$ (or $\Pi_{\psi}^{*}$-full in $X$ ), if the differential on $Q_{(T, F)}(X)$ is zero.

There are various alternative ways to characterize fullness. The inclusion of the fiber $j: X \rightarrow X_{T}$ induces a homomorphism $j^{*}: \Pi_{(T, F)}^{*}(X) \rightarrow \Pi_{\psi}^{*}(X)$; and, in general, $F$ is full if and only if $j^{*}$ is surjective. If $X$ is 1 -connected and of finite type, then it can be shown that $F$ is full if and only if $\Omega X$ is totally nonhomogeneous to zero (with respect to rational (co-)homology) in the fibration $\Omega X \rightarrow(\Omega X)_{T}$ $\rightarrow B_{T}$, where $\Omega X$ is defined using a basepoint in $F$, and $T$ acts on $\Omega X$ in the obvious way. And, under Conditions 1.2(i) and (ii), if $\operatorname{dim}_{Q} \pi_{*}(X) \otimes Q<\infty$, then $F$ is full if and only if $\operatorname{dim}_{Q} \pi_{*}(F) \otimes Q=\operatorname{dim}_{Q} \pi_{*}(X) \otimes Q$ (by [2, Proposition 3.2]).

Thus, if $F$ is full, then $E(T, F ; X)_{1}^{*, *}=E(T, F ; X)_{0}^{*, *}$. We conclude this section by making note of the following. Suppose that $X$ is simply connected. Let $\mathscr{L}_{*}(X)$ be the rational homotopy Lie algebra of $X$. Suppose that $\mathscr{L}_{*}(X)$ is finitely presented, and let $L\left(W_{X}\right) \rightarrow L\left(V_{X}\right) \rightarrow \mathscr{L}_{*}(X) \rightarrow 0$ be a minimal presentation. Let $U=U \mathscr{L}_{*}(X)$ be the universal enveloping algebra of $\mathscr{L}_{*}(X)$. So $U \cong \Omega=$ $H_{*}(\Omega X ; Q)$. (See $[14, \S 5$, for example].) Hence, from Lemaire [9, Chapitre 1], we 
have

$$
\operatorname{dim}_{Q}\left(V_{X}\right)=\operatorname{dim}_{Q} \operatorname{Tor}_{1, *}^{\Omega}(Q, Q)=\operatorname{dim}_{Q} E(X)_{1, *}^{2}
$$

and

$$
\operatorname{dim}_{q}\left(W_{X}\right)=\operatorname{dim}_{Q} \operatorname{Tor}_{2, *}^{\Omega}(Q, Q)=\operatorname{dim}_{Q} E(X)_{2, *}^{2} .
$$

4. Proof of Theorem 1.3 and further results. Before beginning the proof of Theorem 1.3 we note that Conditions 1.1 imply that there is a subcircle $S^{1} \subseteq T$ such that $X^{S^{1}}=X^{T}$. Thus we may assume without loss of generality that $T=S^{1}$, and hence that $R=H^{*}\left(B_{T} ; Q\right)=Q[t]$, the polynomial ring on a single generator $t$ of degree 2. Let $H_{i}=E(T, F ; X)_{2}^{i, *}$, and let $Q_{\alpha}$ for $\alpha \in Q$ indicate $Q$ made into an $R$-module via the map $R \rightarrow Q$ given by $t \rightarrow \alpha$. We shall prove the following refinements of Theorem 1.3:

(1) $\operatorname{dim}_{Q}\left(V_{X}\right)-\operatorname{dim}_{Q}\left(V_{F}\right)=\operatorname{dim}_{Q} \operatorname{Tor}_{1}^{R}\left(H_{2}, Q_{0}\right)$,

(2) $\operatorname{dim}_{Q}\left(W_{X}\right)-\operatorname{dim}_{Q}\left(W_{F}\right)=\operatorname{dim}_{Q} \operatorname{Tor}_{1}^{R}\left(H_{2}, Q_{0}\right)+\operatorname{dim}_{Q} \operatorname{Tor}_{1}^{R}\left(H_{3}, Q_{0}\right)$, and, hence

(3) c.i.d. $\left(\mathscr{L}_{*}(X)\right)-$ c.i.d. $\left(\mathscr{L}_{*}(F)\right)=\operatorname{dim}_{Q} \operatorname{Tor}_{1}^{R}\left(H_{3}, Q_{0}\right)$.

The following simple lemma will be useful.

4.1 Lemma. Let $M$ be a nonnegatively graded R-module. Let $M_{\alpha}=Q_{\alpha} \otimes_{R} M$, and supose that $\operatorname{dim}_{Q} M_{0}<\infty$. Then $M$ is finitely generated, and, for $\alpha \neq 0$,

$$
\operatorname{dim}_{Q}\left(M_{0}\right)=\operatorname{dim}_{Q}\left(M_{\alpha}\right)+\operatorname{dim}_{Q} \operatorname{Tor}_{1}^{R}\left(M, Q_{0}\right) .
$$

Proof. Since $M_{0} \cong M / t M$ and $M$ is nonnegatively graded, it follows that $M$ is finitely generated. Let $0 \rightarrow N \rightarrow L \rightarrow M \rightarrow 0$ be a minimal presentation. Since $\alpha \neq 0$ implies that the functor $Q_{\alpha} \otimes_{R}$ is exact on graded $R$-modules, we have exact sequences

$$
0 \rightarrow \operatorname{Tor}_{1}^{R}\left(M, Q_{0}\right) \rightarrow N_{0} \rightarrow L_{0} \rightarrow M_{0} \rightarrow 0
$$

and

$$
0 \rightarrow N_{\alpha} \rightarrow L_{\alpha} \rightarrow M_{\alpha} \rightarrow 0 .
$$

The result follows since $L$ and $N$ are free.

Proof of Theorem 1.3. Let $\left.C^{p}=E(T, F ; X)\right)^{p * *}=m^{p} / m^{p+1}$, where $m=$ $\operatorname{ker}(\varepsilon(F))$. Thus $C^{*}$ is a complex of free $R$-modules with differential the $d_{1}$ differential of the EMMQSS.

Now for any complex of free $R$-modules $C^{*}$, we have a universal coefficients theorem

$$
0 \rightarrow H^{i}\left(C^{*}\right)_{0} \rightarrow H^{i}\left(C_{0}^{*}\right) \rightarrow \operatorname{Tor}_{1}^{R}\left(H^{i+1}\left(C^{*}\right), Q_{0}\right) \rightarrow 0,
$$

where $C_{0}^{*}=Q_{0} \otimes_{R} C^{*}$, as above.

In our case, however, $C_{0}^{p}=\left(m^{p} / m^{p+1}\right)_{0}=E(X)_{1}^{p, *}$, by the Grivel-HalperinThomas theorem. Let $x_{1}=\operatorname{dim}_{Q}\left(V_{X}\right)$ and $x_{2}=\operatorname{dim}_{Q}\left(W_{X}\right)$. Thus $x_{i}=$ $\operatorname{dim}_{Q} H^{i}\left(C_{0}^{*}\right)$ for $i=1,2$, by the remarks at the end of $\S 3$ and the Felix-HalperinTanré duality. 
So

$$
\begin{aligned}
x_{i}<\infty & \Rightarrow \operatorname{dim}_{Q} H^{i}\left(C^{*}\right)_{0}<\infty \\
& \Rightarrow \operatorname{dim}_{Q} H^{i}\left(C^{*}\right)_{1}<\infty \text { by } 4.1 \\
& \Rightarrow \operatorname{dim}_{Q} E(F)_{2}^{i, *}<\infty \text { by } 3.1,
\end{aligned}
$$

and the fact that $\operatorname{dim}_{Q}\left(Q_{1} \otimes_{R} M\right)=\operatorname{dim}_{R_{(0)}}\left(R_{(0)} \otimes_{R} M\right)$ for any finitely generated $R$-module $M$. Thus $\mathscr{L}_{*}(F)$ is finitely presented by Lemaire [9, 1.1.11].

Now let $f_{1}=\operatorname{dim}_{Q}\left(V_{F}\right)$ and $f_{2}=\operatorname{dim}_{Q}\left(W_{F}\right)$. Then

$$
\begin{aligned}
f_{i} & =\operatorname{dim}_{Q} E(F)_{2}^{i, *}=\operatorname{dim}_{Q} H^{i}\left(C^{*}\right)_{1} \\
& =\operatorname{dim}_{Q} H^{i}\left(C^{*}\right)_{0}-\operatorname{dim}_{Q} \operatorname{Tor}_{1}^{R}\left(H^{i}\left(C^{*}\right), Q_{0}\right) \\
& =\operatorname{dim}_{Q} H^{i}\left(C_{0}^{*}\right)-\operatorname{dim}_{Q} \operatorname{Tor}_{1}^{R}\left(H^{i+1}\left(C^{*}\right), Q_{0}\right)-\operatorname{dim}_{Q} \operatorname{Tor}_{1}^{R}\left(H^{i}\left(C^{*}\right), Q_{0}\right),
\end{aligned}
$$

by 4.1 and the universal coefficients theorem.

Since $R=Q[t]$ is a principal ideal domain, and since $d: C^{0}=R \rightarrow C^{1}$ is zero, $H^{1}\left(C^{*}\right)$ is a free $R$-module; and so $\operatorname{Tor}_{1}^{R}\left(H^{1}\left(C^{*}\right), Q_{0}\right)=0$. Thus the proof is finished.

4.2 REMARK. Since $\mathbf{Z}_{2}$-grading is preserved in the proof of 1.3 , the statement can be refined into separate statements about generators, relations, and c.i.d. of even degree and odd degree.

Next we give a simple corollary, and then two examples to show that the fullness condition on $F$ is needed in Theorem 1.3.

4.3 Corollary. If $X$ and $F$ satisfy Conditions 1.1 and 1.2 , and if $\mathscr{L}_{*}(X)$ is a free Lie algebra on $n_{0}$ generators of even degree and $n_{1}$ generators of odd degree, then $\mathscr{L}_{*}(F)$ is also a free Lie algebra on $n_{0}$ generators of even degree and $n_{1}$ generators of odd degree.

Proof. With the notation of the proof of 1.3 , we have $x_{2}=0$. Hence $f_{2}=0$ and $\operatorname{dim}_{Q} \operatorname{Tor}_{1}^{R}\left(H^{2}\left(C^{*}\right), Q_{0}\right)=0$. So $\mathscr{L}_{*}(F)$ is free, and $f_{1}=x_{1}$.

4.4 Example. Let $R=Q[t], \operatorname{deg}(t)=2$. Consider the homomorphism of $R$-algebras $\varphi: R\left[X_{1}, X_{2}, X_{3}\right] / I \rightarrow R\left[u_{1}, u_{2}\right] / J$, where $I$ is the ideal $\left(X_{2}^{2}-t^{2} X_{3}, X_{1} X_{3}, X_{1}^{4}\right.$ $\left.-X_{3}^{2}\right), \quad J=\left(u_{1}^{2} u_{2}, u_{1}^{4}-u_{2}^{4}\right), \operatorname{deg}\left(X_{1}\right)=4, \operatorname{deg}\left(X_{2}\right)=6, \operatorname{deg}\left(X_{3}\right)=8, \operatorname{deg}\left(u_{1}\right)=$ $\operatorname{deg}\left(u_{2}\right)=2$, and $\varphi$ is given by $\varphi\left(X_{1}\right)=t u_{2}, \varphi\left(X_{2}\right)=t^{2} u_{1}$, and $\varphi\left(X_{3}\right)=t^{2} u_{1}^{2}$.

By [12], $\varphi$ may be formally realized as the map $H_{T}^{*}(X ; Q) \rightarrow H_{T}^{*}(F ; Q)$ for some $T=S^{1}$-action on a space $X$ with connected fixed point set $F$. $F$, however, is not full; and, calculating by the methods above, we find that $x_{1}=3, x_{2}=4$, c.i.d. $\left(\mathscr{L}_{*}(X)\right)=1, f_{1}=4, f_{2}=8$, and c.i.d. $\left(\mathscr{L}_{*}(F)\right)=4$. Thus parts (1)-(3) of Theorem 1.3 are violated.

4.5 EXAmple. Let $Z$ be a finite 1 -connected $C W$-complex such that $\mathscr{L}_{*}(Z)$ is either not finitely generated or finitely generated but not finitely presented. (See [9, §3.3] for a construction of such spaces.) Let $X=\Sigma^{2} Z$, the double suspension of $Z$. Then $\mathscr{L}_{*}(X)=L\left(\tilde{H}_{*}(\Sigma Z ; Q)\right)$ by the Bott-Samelson theorem. Now there is an action of $T=S^{1}$ on $X$ with $X^{T}=Z$. Thus the part of Theorem 1.3 concerning the inheritance of finite presentations can fail if $F$ is not full. 
We conclude with a result on iterated rational Whitehead products and degrees of nilpotency. Let $L$ be a Lie algebra (rational and positively graded in our case) and let $\beta: L \otimes L \rightarrow L$ denote the bracket: That is $\beta(x \otimes y)=[x, y]$. Then the iterated bracket map of length $q$ may be defined inductively as

$$
\beta(q)=\beta(I \otimes \beta(q-1)): \otimes{ }^{q} L=L \otimes \otimes^{q-1} L \rightarrow L \otimes L \rightarrow L,
$$

where $\beta(2)=\beta$. Dually, for a co-Lie algebra $\mathscr{L}$ with cobracket $\nabla: \mathscr{L} \rightarrow \mathscr{L} \otimes \mathscr{L}$,

$$
\nabla(q)=(I \otimes \nabla(q-1)) \nabla: \mathscr{L} \rightarrow \mathscr{L} \otimes \mathscr{L} \rightarrow \mathscr{L} \otimes \otimes^{q-1} \mathscr{L}=\otimes^{q} \mathscr{L},
$$

where $\nabla(2)=\nabla$. Let $C^{q} L$ denote the image of $\beta(q)$, the ideal of iterated brackets of length $q$. Then the quotient $L / C^{q} L=\operatorname{coker} \beta(q)$ corresponds under duality to $\operatorname{ker} \nabla(q)$.

4.6 THEOREM. Let a torus $T$ act on a space $X$ with nonempty fixed point set, and let $F$ be a component of the fixed point set. Suppose that $X$ and $F$ satisfy Conditions (1.1) and (1.2). If, for some $q \geqslant 2, \operatorname{dim}_{Q} \mathscr{L}_{*}(X) / C^{q} \mathscr{L}_{*}(X)<\infty$, then

$$
\operatorname{dim}_{Q} \mathscr{L}_{*}(F) / C^{q} \mathscr{L}_{*}(F)<\infty
$$

and

$$
\operatorname{dim}_{Q} \mathscr{L}_{*}(F) / C^{q} \mathscr{L}_{*}(F) \leqslant \operatorname{dim}_{Q} \mathscr{L}_{*}(X) / C^{q} \mathscr{L}_{*}(X) .
$$

In particular, if $\operatorname{dim}_{Q} \mathscr{L}_{*}(X)<\infty$, then

$$
\operatorname{dim}_{Q} C^{q} \mathscr{L}_{*}(F) \geqslant \operatorname{dim}_{Q} C^{q} \mathscr{L}_{*}(X) .
$$

(Thus degree of nilpotency of $\mathscr{L}_{*}(F) \geqslant$ degree of nilpotency of $\mathscr{L}_{*}(X)$.)

Proof. As before, we may assume that $T=S^{1}$. Since $F$ is full, using the notation above (e.g., $m=\operatorname{ker} \varepsilon(F))$, by $[2, \S 5]$ we have an $R$-co-Lie algebra structure $\nabla$ : $\mathrm{m} / \mathrm{m}^{2} \rightarrow \mathrm{m} / \mathrm{m}^{2} \otimes \mathrm{m} / \mathrm{m}^{2}$, which, when evaluated at $t=0$ and $t=1$, yields the co-Lie-algebra structures on $m(X) / m(X)^{2}$ and $m(F) / m(F)^{2}$, respectively, which are dual to the Lie algebra structures on $\mathscr{L}_{*}(X)$ and $\mathscr{L}_{*}(F)$, respectively. (Strictly speaking, $m / m^{2}, m(X) / m(X)^{2}$, and $m(F) / m(F)^{2}$ need to have their degrees lowered by one when they are to be regarded as co-Lie-algebras.) We may form the iterate $\nabla(q)$ as above, and the result follows because $\operatorname{dim}_{Q} \operatorname{ker}\left(\nabla(q) \otimes_{R} Q_{1}\right)=$ $\operatorname{dim}_{Q}(\operatorname{ker} \nabla(q))_{1}$, by exactness of $\otimes_{R} Q_{1}, \operatorname{dim}_{Q}(\operatorname{ker} \nabla(q))_{1}=\operatorname{dim}_{Q}(\operatorname{ker} \nabla(q))_{0}$, since $m / m^{2}$ is a free $R$-module, and $\operatorname{dim}_{Q}(\operatorname{ker} \nabla(q))_{0} \leqslant \operatorname{dim}_{Q} \operatorname{ker}\left(\nabla(q) \otimes_{R} Q_{0}\right)$, since $\otimes{ }^{a} \mathrm{~m} / \mathrm{m}^{2}$ is a free $R$-module. The last statement follows since fullness implies that $\operatorname{dim}_{Q} \mathscr{L}_{*}(F)=\operatorname{dim}_{Q} \mathscr{L}_{*}(X)$.

4.7 Remarks. (i) Again we may refine the statements of Theorem 4.6 into separate statements about elements of even degree and elements of odd degree.

(ii) If we assume only Conditions 1.1 and that $X$ is TNHZ, then 4.6 has a cohomology analogue concerning products of length $q$ or more and cup length. This follows from 4.1 applied to iterates of the cup product map

$$
\mu: \tilde{H}_{T}^{*}\left(X, x_{0} ; Q\right) \otimes \tilde{H}_{T}^{*}\left(X, x_{0} ; Q\right) \rightarrow \tilde{H}_{T}^{*}\left(X, x_{0} ; Q\right),
$$

where $x_{0}$ is basepoint in $F$. It follows that

$$
\operatorname{dim}_{Q} \tilde{H}^{*}(F ; Q) /\left[\tilde{H}^{*}(F ; Q)\right]^{q} \leqslant \operatorname{dim}_{Q} \tilde{H}^{*}(X ; Q) /\left[\tilde{H}^{*}(X ; Q)\right]^{q}
$$


for $q \geqslant 0$. Hence if $X^{T}=F$ is connected, $n_{0}(F) \geqslant n_{0}(X)$, where $n_{0}$ is the rational cup length: That is $n_{0}(X)=\max \left\{q:\left[\tilde{H}^{*}(X ; Q)\right]^{q} \neq 0\right\}$ (cf. [7, §9]). There is an example due to Tomter [17] where $X=S^{4} \times S^{2 n}$ and $F=C P^{3} ; n_{0}(F)>n_{0}(X)$ in this example.

\section{REFERENCES}

1. C. J. Allday, On the rational homotopy of fixed point sets of torus actions, Topology 17 (1978), $95-100$.

2. __ Rational homotopy and torus actions, Houston J. Math. 5 (1979), 1-19.

3. C. J. Allday and S. Halperin, Sullivan-de Rham theory for rational Alexander-Spanier cohomology, Houston J. Math. 10 (1984), 15-33.

4. C. J. Allday and V. Puppe, On the rational homotopy of circle actions (Proc. Aarhus Sympos. on Algebraic Topology, 1982), Lecture Notes in Math., vol. 1051, Springer-Verlag, Berlin and New York, 1984.

5. H. J. Baues and J. M. Lemaire; Minimal models in homotopy theory, Math. Ann. 225 (1977), $219-242$.

6. T. Chang, On the number of relations in the cohomology of a fixed point set, Manuscripta Math. 18 (1976), 237-247.

7. Y. Felix and S. Halperin, Rational L.-S. category and its applications, Publ. IRMA Lille (1980).

8. S. Halperin, Lectures on minimal models, Publ. Internes U.E.R. Math. Pures et Appl., No. 111, Univ. des Sciences et Techniques de Lille I, 1977.

9. J. M. Lemaire, Algèbres connexes et homologie des espaces de lacets, Lecture Notes in Math., vol. 422, Springer-Verlag, Berlin and New York, 1974.

10. J. C. Moore, Algèbre homologique et homologie des espaces classifiants, Séminaire H. Cartan 1959/60, Exposé 7.

11. V. Puppe, Cohomology of fixed point sets and deformation of algebras, Manuscripta Math. 23 (1978), $343-354$.

12. $119-136$ . Deformation of algebras and cohomology of fixed point sets, Manuscripta Math. 30 (1979),

13. , P. A. Smith theory via deformations, Homotopie Algébrique et Algèbre Locale, Astérisque, 1984.

14. D. Quillen, Rational homotopy theory, Ann. of Math. (2) 90 (1969), 205-295.

15. D. Sullivan, Infinitesimal computations in topology, Inst. Hautes Ėtudes Sci. Publ. Math. 47 (1977), 269-332.

16. D. Tantré, Dualité d'Eckmann-Hilton à travers les modèles de Chen-Quillen-Sullivan, Cahiers Topologie Géom. Différentielle 22 (1981), 53-60.

17. P. Tomter, Transformation groups on cohomology products of spheres, Invent. Math. 23 (1974), $79-88$.

Department of Mathematics, University of Hawail, Honolulu, Hawail 96822

FakUltät fUr Mathematik, Univerisität Konstanz, D - 7750 Konstanz 1, West Germany 